\title{
Effect of Lactobacillus brevis SBC8803 on Gamma-Glutamyl Transferase in Japanese Habitual Drinkers: A Double-Blind, Placebo-Controlled Study
}

\author{
Yoshihisa Wakita $^{1 *}$, Hajime Kanda ${ }^{1}$, Chikako Shimizu ${ }^{2}$, Yasukazu Nakakita ${ }^{1}$, Hirotaka Kaneda ${ }^{2}$, \\ Shuichi Segawa ${ }^{1}$, Moeko Ozaki ${ }^{1}$, Tatsuro Shigyo ${ }^{1}$, Takaaki Ohtake ${ }^{3}$, Mikihiro Fujiya ${ }^{3}$, Yutaka Kohgo ${ }^{3}$ \\ ${ }^{1}$ Frontier Laboratories of Value Creation, Sapporo Breweries Ltd., Shizuoka, Japan; ${ }^{2}$ Corporate Planning Department, Sapporo \\ Holdings Ltd., Tokyo, Japan; ${ }^{3}$ Division of Gastroenterology and Hematology/Oncology, Department of Medicine, Asahikawa Medi- \\ cal University, Hokkaido, Japan. \\ Email: *yoshihisa.wakita@sapporobeer.co.jp
}

Received February 20 $0^{\text {th }}, 2012$; revised March $30^{\text {th }}$, 2012; accepted April $8^{\text {th }}, 2012$

\begin{abstract}
A randomized, double-blind, placebo-controlled clinical trial in Japanese habitual drinkers was conducted to evaluate the efficacy of Lactobacillus brevis SBC8803 to alleviate adverse effect of alcohol. Subjects who drank habitually and had moderately higher levels of gamma-glutamyl transferase (GGT) (50 - $100 \mathrm{IU} / \mathrm{L})$ were enrolled. The levels of transaminases in these subjects were almost within normal levels (aspartate transaminase (AST) $<30 \mathrm{IU} / \mathrm{L}$ and alanine transaminase (ALT) $<40 \mathrm{IU} / \mathrm{L})$. Either the capsules containing placebo $(\mathrm{n}=23)$ or $130 \mathrm{mg}\left(4.0 \times 10^{10}\right.$ colony-forming units) of live L. brevis SBC8803 $(\mathrm{n}=22)$ per day were administered for the continuous eight weeks (56 days). During the period, the subjects both in test group and placebo groups have kept each drinking behavior as usual. Regarding lipid metabolism, triacylglycerol (TG) levels in the male test group significantly decreased at week 4 as compared with week 0. Biomarkers of hepatocytes-damage; AST and ALT levels showed no significant differences between the placebo and test groups at both weeks 4 and 8. Oxidative stress marker; GGT at weeks 4 was significantly lower in the test group than that in the placebo group ( $\mathrm{p}=0.017)$, but not at weeks 8 . However, taking a reduced rate of GGT at weeks 8 comparing with that at week 0 , that in the test group showed larger value comparing with that in the placebo group. These data about TG and GGT suggest that, although efficacy of L. brevis SBC8803 is limited in this study, intake of the probiotic may alleviate alcoholic influence in lipid metabolism and oxidative stress.
\end{abstract}

Keywords: Lactobacillus brevis; Gamma-Glutamyl Transferase; Alcoholic Related Disorder; Double-Blind Placebo-Controlled Study

\section{Introduction}

The harmful use of alcohol is a worldwide problem resulting in millions of death [1]. It is not only a causal factor in many diseases, but also a precursor to injury and violence. In Japan, recent estimates suggest that $36 \%$ of adult males and $6.4 \%$ of adult females consume alcohol [2], and $14.7 \%$ of out-patients in clinics are considered to be somewhat alcohol-related [3]. One of the best-known target organs is liver, which is eventually damaged by chronic alcohol consumption and resulted in fatty liver, hepatitis, and cirrhosis [4]. As these diseased hepatic conditions are hazardous for human health and socioeconomic societies, the prevention to develop such conditions is one of the most important issues for habitual drinkers. It is known that biochemical parameters such as

${ }^{*}$ Corresponding author.
GGT and TG are increased in serum prior to the development of alcoholic liver diseases, and these parameters are frequently used for monitoring the alcohol consumption and the prediction on the initial manifestation of liver damage [5].

Concerning the increase of GGT, a couple of mechanisms have been postulated. Up until recently, the increase of GGT is chiefly induced by alcohol itself, because this increase is observed in subjects who have not developed alcoholic liver diseases. In both alcoholic and non-alcoholic fatty liver, the increase of GGT was known well and this phenomenon was not considered to be meaningful for further development of these diseases [6]. However, the meaning of elevated GGT is getting more important than thought to be. In the clinical standpoints of view, the risks of future developments of liver diseases, diabetes, and cardiovascular disease are increased 
in several cohort studies when GGT was high $[7,8]$. In addition, from the biochemical points, the increase of GGT was resulted by the increase of oxidative stresses [9], and the initial manifestation of the oxidative stressinduced diseased conditions including alcoholic liver injury, and non-alcoholic steatohepatitis in patients with metabolic syndrome [10]. Therefore, it is getting more important to monitor GGT as the initial manifestation of alcohol-induced organ injury, in addition to monitoring marker for chronic alcohol consumption.

It is well known that many subjects who habitually have drunk alcohol are suffered from biological effects of chronic alcohol consumption and the production of fatty liver, which is characterized by the accumulation of TG and cholesterol in hepatocytes. The accumulation of TG within the liver is considered to be an early and reversible effect of alcohol consumption. As alcohol increases peripheral lipolysis and alters liver redox potential, which increases fatty acid synthesis, the subsequent increase of fatty acids esterification, will cause TG accumulation [4].

Probiotics are defined as live microorganisms that confer a health benefit on the host. Human data suggests an emerging role for probiotic therapy in the treatment of liver diseases, including alcoholic liver disease [11,12]. We have reported that Lactobacillus brevis SBC8803 ameliorates ethanol-induced liver injury and fatty liver in C57BL/6N mice fed an ethanol-containing diet [13]. We think that there is a meaningful strategy to alleviate adverse influence of alcohol by the dietary intake of probiotic bacteria. Therefore, in this study, we evaluated the efficacy of $L$. brevis SBC8803 to alleviate adverse effect of alcohol in habitual drinkers whose serum GGT are slightly high levels (50 - 100 IU/L) and, AST and ALT levels are within normal range.

\section{Methods}

\subsection{Subjects}

Study subjects were recruited from Ueno Clinic Co., LTA, and the Tokyo Clinical Center in Tokyo, Japan. Selection criteria included subjects between 20 and 64 years with GGT concentrations between 50 and $100 \mathrm{IU} / \mathrm{L}$ and who drank habitually. The upper limit of normal in Japanese adult is $50 \mathrm{IU} / \mathrm{L}$ in most medical facilities. Subjects were excluded if they were positive for HBs antigen or HCV antibody. Clinical screening (fasting blood tests, urine samples, and medical history) and a dietary questionnaire were further used to select subjects. Sixty-four subjects were selected to participate in this clinical trial and randomly assigned to either the test or placebo group. All subjects were employed Japanese individuals who followed a daily routine. The study was performed in accordance with the Declaration of Helsinki and regulations of the Ueno Clinic ethics committee for Internal Clinical Studies. All subjects provided written informed consent before participating in the study.

\subsection{Test Capsules}

The components of the placebo and test sample capsules were shown in Table 1. Twelve test capsules contained $4.0 \times 10^{10}$ colony-forming units (CFUs) $\left(3.3 \times 10^{9} \mathrm{CFU} /\right.$ capsule) of $L$. brevis SBC8803. Placebo and test capsules could not be distinguished from each other with the naked eye. These capsules were kept in refrigerators in the subject's house.

\subsection{Protocol}

Subjects consumed twelve placebo or test capsules daily for eight weeks. These capsules were consumed after one of their three daily meals (breakfast, lunch, or dinner). Subjects maintained their usual alcohol drinking habits throughout the study. Subjects also completed a questionnaire, wherein they specified the quantity and time of capsule intake, daily drinking, and a detailed dietary record for three days before clinical examinations. Data of a subject was excluded if the subject consumed less than one-third or more than three times their weekly preobservation alcohol intake level during the study.

\subsection{Blood Chemistry Analyses}

Blood samples were collected from subjects' forearms every four weeks at the clinic using blood collection tubes that did not contain anticoagulants. Participants were instructed to abstain from food and beverages, except water, for $12 \mathrm{~h}$ before each of their clinical exami-

Table 1. Placebo and test capsule composition (per 12 capsules).

\begin{tabular}{ccc}
\hline Constituents (mg) & Placebo capsule & Test capsule \\
\hline L. brevis SBC8803 $^{\mathrm{a}}$ & 0 & 130 \\
Maltitol & 112 & 112 \\
Sucrose & 48 & 48 \\
Cellulose & 1738 & 1738 \\
Calcium stearate & 120 & 120 \\
Highly dispersed silicon dioxide & 43 & 43 \\
Starch hydrolysate & 339 & 137 \\
Dextrose & 0 & 72 \\
Total & 2400 & 2400 \\
\hline
\end{tabular}

${ }^{\mathrm{a}}$ Twelve test capsules contained $4.0 \times 10^{10} \mathrm{CFUs}\left(3.3 \times 10^{9} \mathrm{CFU} /\right.$ capsule $)$ of L. brevis SBC8803. 
nations. The blood samples were analyzed by Mitsubishi Chemical Medicine Ltd., Tokyo, Japan. Serum GGT, AST, ALT, and TG levels were determined using a biochemical analyzer (Hitachi 7600-300S, Hitachi Ltd., Tokyo, Japan).

\subsection{Statistical Analyses}

Statistical analyses were performed for the forty-five subjects (placebo group: $n=23$; test group: $n=22$ ) who completed the examination. Subject mean ages for the placebo and test groups were $43.9 \pm 5.2$ and $43.0 \pm 7.0$ years, respectively. Data was analyzed using the SPSS software $13.0 \mathrm{~J}$ for Windows (SPSS Inc., Japan) and is presented as the mean \pm standard deviation (SD). Analysis of covariance was applied to primary outcome (measured serum GGT) data. Between-group p values were calculated by analysis of covariance with adjustment for baseline values. Secondary outcome (AST, ALT, and TG) absolute values were determined at weeks 4 and 8 , and data was compared using unpaired t-tests. Changes from baseline values were calculated for the test and placebo groups, and differences were evaluated using t-tests. In all analyses, a two-sided p value of 0.05 was considered statistically significant.

\section{Results}

\subsection{Subjects}

The flow of study subjects is illustrated in Figure 1. One of the sixty-four subjects dropped out of the trial due to hospital admission. Eighteen subjects (nine from each group) were excluded. Details for the excluded subjects were as follows: The average weekly alcohol intake of six subjects (three from each group) was three times or more than pre-observation levels. Five subjects (two and three from the placebo and test groups, respectively) drank one-third or less than pre-observation levels. Five subjects gained weight $(>3 \mathrm{~kg}$ ) because of a change in lifestyle such as quitting smoking and exercise, and consuming heavy meals. One subject (placebo group) showed high TG levels (1354 mg/dL) during week 8 and could not come to the clinic for re-examination. Another subject consumed a large quantity of medicine for cold symptoms. The characteristics of the subjects at the base line are listed in Table 2. There were no significant differences between placebo and test groups with regard

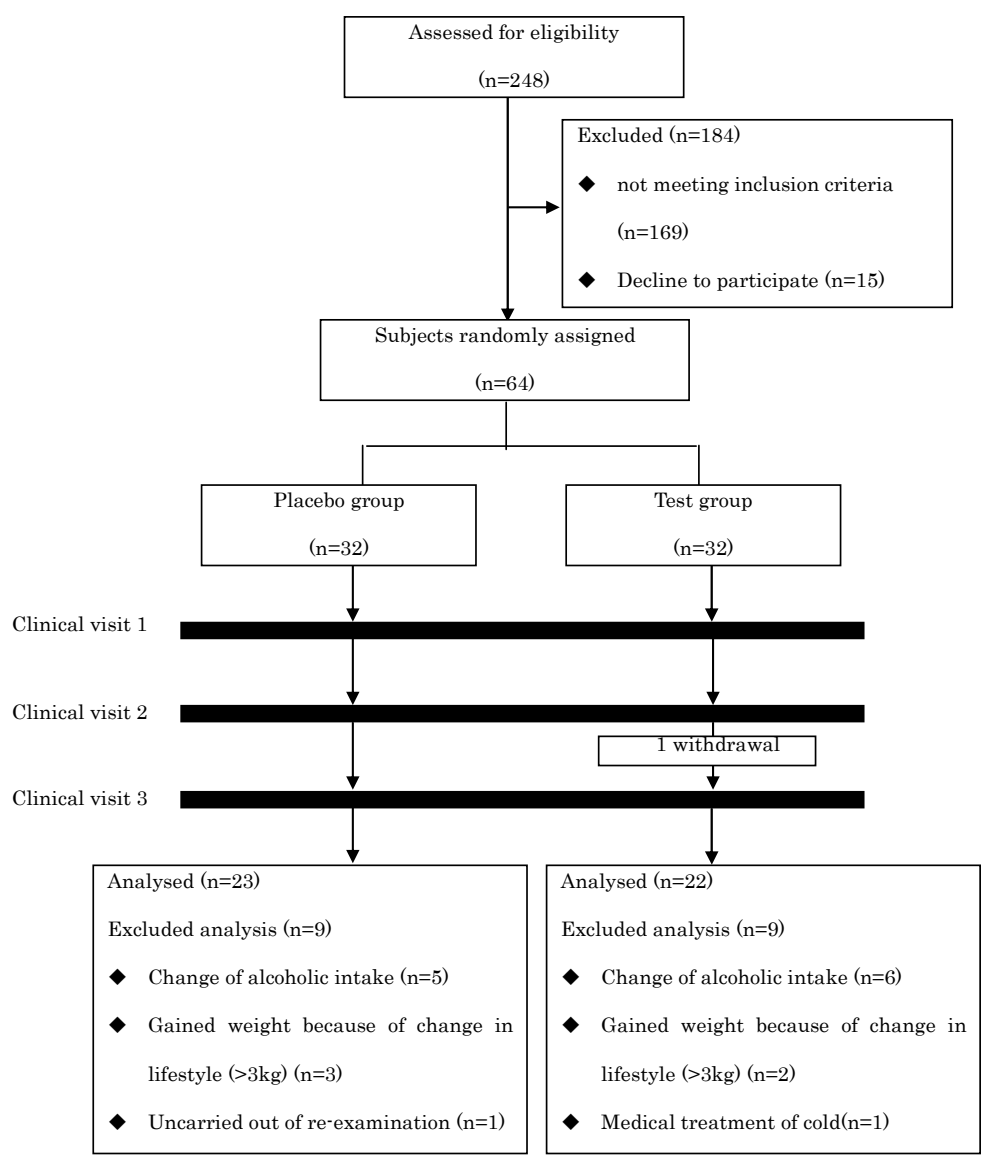

Figure 1. Flow of subjects through the trial. 
Table 2. Characteristics of subjects at baseline (values are mean \pm standard deviation).

\begin{tabular}{cccc}
\hline & Placebo group & Test group & $P$ at baseline \\
\hline Male/female & $17 / 6$ & $17 / 5$ & - \\
Age (year) & $43.9 \pm 5.2$ & $43.0 \pm 7.0$ & 0.655 \\
Body weight (kg) & $67.6 \pm 7.9$ & $69.4 \pm 11.0$ & 0.593 \\
BMI $^{\mathrm{a}}\left(\mathrm{kg} / \mathrm{m}^{2}\right)$ & $23.7 \pm 2.9$ & $24.3 \pm 3.1$ & 0.508 \\
GGT (IU/L) & $66.1 \pm 20.1$ & $73.7 \pm 21.8$ & 0.231 \\
AST (IU/L) & $22.7 \pm 5.8$ & $21.8 \pm 3.8$ & 0.512 \\
ALT (IU/L) & $23.7 \pm 9.4$ & $24.4 \pm 8.1$ & 0.787 \\
TG (mg/dL) & $114.7 \pm 84.6$ & $145.0 \pm 95.8$ & 0.267 \\
\hline
\end{tabular}

${ }^{a}$ BMI, body mass index. Capsules containing placebo $(\mathrm{n}=23)$ or $130 \mathrm{mg}$ $\left(4.0 \times 10^{10}\right.$ colony-forming units) of live $L$. brevis SBC8803 $(\mathrm{n}=22)$ per day were administered for the continuous eight weeks (56 days).

to age, body weight, body mass index, serum GGT, AST, ALT, and TG.

\subsection{Capsule Consumption and Alcohol and Dietary Intake}

Placebo and test capsule consumption during the trial was $>95 \%$. No significant differences were observed between the groups for intake of alcohol (Table 3). There were no significant differences for calorie intake between the placebo (week 4, $2070 \pm 603 \mathrm{kcal} /$ day; week $8,2116 \pm 562 \mathrm{kcal} /$ day) and test groups (week 4, $2237 \pm$ $486 \mathrm{kcal} /$ day; week 8, $2194 \pm 546 \mathrm{kcal} /$ day).

\subsection{Blood Chemistry}

GGT, AST, ALT, and TG concentrations are listed in Table 4. At week 4, subjects in the test group showed significantly lower GGT levels as compared with those in the placebo group $(p=0.017)$. Although no significant changes in GGT levels were observed between the test and placebo groups at week 8, GGT levels in the placebo group were significantly elevated from week 0 , while those in the test group remained unchanged. No significant differences were observed for AST, ALT, and TG levels between the placebo and test groups at weeks 4 and 8.

\section{Discussion}

Chronic and large amount of alcohol consumption significantly correlates detrimental effects as alcohol-related diseases; psychological problems, malnutrition, diseases of central nervous system and peripheral nervous system, cardiovascular disease, liver dysfunction, pancreatitis, increased risk of type 2 diabetes, cancers of head, neck, esophagus, colon and liver. Long-term use of alcohol in excessive quantities is capable of damaging nearly every organ and system in the body both of directly and indirectly. Therefore indirect mechanism of organ damages might be caused by oxidized stress by metabolic changes by alcohol.

During the initial stages of alcoholic liver disease, TG accumulates in hepatocytes leading to the development of steatosis, which is a reversible condition. If alcohol consumption is continued, steatosis can progress to severe fatty liver, fibrosis, cirrhosis, and even hepatocellular carcinoma, particularly in the presence of other comorbid factors [14].

Some effects of probiotics on liver function have been reported. Kirpich et al. showed that short-term oral supplementation with Bifidobacterium bifidum and Lactobacillus plantarum 8PA3 was associated with the restoration of bowel flora and greater recovery from alcohol-induced liver injury than standard therapy alone [11]. However, the early intervention with probiotics is expected to prevent the development of alcoholic liver disease. In this study, we investigate the effect of $L$. brevis in Japanese habitual drinkers without obvious hepatocytes damage by alcohol. They were relatively healthy subjects whose GGT values are from upper limit of normal to 2-fold (50 - $100 \mathrm{IU} / \mathrm{L}$ ) and, AST and ALT levels are within normal range.

To the best of our knowledge, this is the first investigation regarding the role of probiotics in the improvement of serum GGT for habitual drinkers based on a double-blind, placebo-controlled study. GGT, a wellknown biomarker for alcoholic liver damage, is present in hepatocytes and biliary epithelial cells. Although elevated GGT levels initially result from enzyme induction

Table 3. Intake of alcohol in the placebo and test groups (values are mean \pm standard deviation).

\begin{tabular}{cccccccccc}
\hline \multicolumn{10}{c}{ Intake of alcohol (g/day) } \\
\cline { 2 - 10 } & Preobservation (0w) & $1 \mathrm{w}$ & $2 \mathrm{w}$ & $3 \mathrm{w}$ & $4 \mathrm{w}$ & $5 \mathrm{w}$ & $6 \mathrm{w}$ & $7 \mathrm{w}$ & $8 \mathrm{w}$ \\
\hline Placebo group & $52.2 \pm 28.1$ & $45.2 \pm 26.5^{* * *}$ & $44.7 \pm 27.4^{*}$ & $44.3 \pm 27.3^{*}$ & $43.7 \pm 29.9^{* * *}$ & $43.9 \pm 28.2^{*}$ & $46.4 \pm 26.3$ & $46.3 \pm 32.7$ & $45.9 \pm 30.3$ \\
Test group & $50.6 \pm 31.1$ & $47.3 \pm 32.2$ & $50.9 \pm 36.7$ & $50.8 \pm 38.5$ & $49.3 \pm 41.0$ & $41.7 \pm 24.1^{* *}$ & $42.9 \pm 23.6^{*}$ & $41.0 \pm 23.3^{*}$ & $44.7 \pm 24.2$ \\
\hline
\end{tabular}

${ }^{*} \mathrm{p}<0.05 ;{ }^{* *} \mathrm{p}<0.01$, changes from pre-observation by $t$-test. 
Table 4. Baseline GGT, AST, ALT, and TG levels in the placebo and test groups (values are mean \pm standard deviation).

\begin{tabular}{|c|c|c|c|c|c|}
\hline & & & Baseline & Week 4 & Week 8 \\
\hline \multirow{8}{*}{$\begin{array}{c}\text { All subjects } \\
\text { (Placebo, } \mathrm{n}=23 \text {; Test, } \mathrm{n}=22 \text { ) }\end{array}$} & \multirow{2}{*}{ GGT (IU/L) } & Placebo & $66.1 \pm 20.1$ & $76.5 \pm 48.4^{\dagger}$ & $76.7 \pm 36.1^{*}$ \\
\hline & & Test & $73.7 \pm 21.8$ & $69.3 \pm 27.0$ & $78.6 \pm 34.7$ \\
\hline & \multirow{2}{*}{ AST (IU/L) } & Placebo & $22.7 \pm 5.8$ & $24.8 \pm 6.9$ & $25.7 \pm 7.7^{*}$ \\
\hline & & Test & $21.8 \pm 3.8$ & $24.0 \pm 4.3^{* *}$ & $24.5 \pm 4.2^{* *}$ \\
\hline & \multirow{2}{*}{ ALT (IU/L) } & Placebo & $23.7 \pm 9.4$ & $25.1 \pm 11.5$ & $26.3 \pm 9.9$ \\
\hline & & Test & $24.4 \pm 8.1$ & $25.9 \pm 9.3$ & $27.7 \pm 10.1^{*}$ \\
\hline & \multirow{2}{*}{ TG (mg/dL) } & Placebo & $114.7 \pm 84.6$ & $94.6 \pm 44.4$ & $116.4 \pm 60.8$ \\
\hline & & Test & $145.0 \pm 95.8$ & $111.0 \pm 57.0$ & $122.5 \pm 70.8$ \\
\hline \multirow{8}{*}{$\begin{array}{c}\text { Male subjects } \\
\text { (Placebo, } \mathrm{n}=17 ; \text { Test, } \mathrm{n}=17 \text { ) }\end{array}$} & \multirow{2}{*}{ GGT (IU/L) } & Placebo & $70.9 \pm 20.9$ & $83.5 \pm 54.8^{\dagger}$ & $82.8 \pm 40.0$ \\
\hline & & Test & $72.8 \pm 17.4$ & $68.0 \pm 23.2$ & $76.7 \pm 29.1$ \\
\hline & \multirow{2}{*}{ AST (IU/L) } & Placebo & $21.8 \pm 5.1$ & $24.9 \pm 7.3^{*}$ & $24.8 \pm 6.9^{*}$ \\
\hline & & Test & $22.2 \pm 3.9$ & $23.9 \pm 4.0$ & $24.9 \pm 4.0^{*}$ \\
\hline & \multirow{2}{*}{ ALT (IU/L) } & Placebo & $24.5 \pm 10.0$ & $26.7 \pm 12.2$ & $27.5 \pm 10.5$ \\
\hline & & Test & $26.8 \pm 7.6$ & $27.0 \pm 9.7$ & $29.6 \pm 9.6$ \\
\hline & \multirow{2}{*}{$\mathrm{TG}(\mathrm{mg} / \mathrm{dL})$} & Placebo & $123.6 \pm 97.1$ & $100.6 \pm 48.8$ & $111.3 \pm 58.6$ \\
\hline & & Test & $163.0 \pm 98.9$ & $115.5 \pm 58.3^{*}$ & $130.5 \pm 72.8$ \\
\hline \multirow{8}{*}{$\begin{array}{c}\text { Female subjects } \\
\text { (Placebo, } n=6 \text {; Test, } n=5 \text { ) }\end{array}$} & \multirow{2}{*}{ GGT (IU/L) } & Placebo & $52.7 \pm 9.6$ & $56.8 \pm 7.4$ & $59.3 \pm 10.9$ \\
\hline & & Test & $76.8 \pm 35.5$ & $73.6 \pm 40.3$ & $85.2 \pm 53.4$ \\
\hline & \multirow{2}{*}{ AST (IU/L) } & Placebo & $25.3 \pm 7.4$ & $24.5 \pm 6.4$ & $28.2 \pm 9.9$ \\
\hline & & Test & $20.2 \pm 3.1$ & $24.6 \pm 5.7$ & $22.8 \pm 5.0$ \\
\hline & \multirow{2}{*}{ ALT (IU/L) } & Placebo & $21.3 \pm 8.0$ & $20.7 \pm 8.5$ & $23.0 \pm 7.9$ \\
\hline & & Test & $16.4 \pm 2.9$ & $22.2 \pm 7.6$ & $21.4 \pm 10.1$ \\
\hline & \multirow{2}{*}{$\mathrm{TG}(\mathrm{mg} / \mathrm{dL})$} & Placebo & $89.5 \pm 15.9$ & $77.3 \pm 24.0$ & $131.0 \pm 70.3$ \\
\hline & & Test & $83.6 \pm 54.3$ & $95.4 \pm 55.7$ & $95.2 \pm 62.6$ \\
\hline
\end{tabular}

${ }^{\dagger} \mathrm{p}<0.05$. Analysis of covariance was applied for primary outcomes (measured serum GGT) between the two groups. To compare secondary outcomes (AST, ALT, and TG) between the two groups, unpaired $t$-tests were used. ${ }^{*} \mathrm{p}<0.05,{ }^{* *} \mathrm{p}<0.01$, changes from baselines by $t$-test.

by alcohol and not from hepatic damage, a continual increase in GGT generally indicates that the liver is being damaged. In this study, subjects showed normal AST and ALT levels, indicating that the liver of these subjects is not levels that would indicate alcoholic liver disease. We assumed that the administration of L. brevis SBC8803 would help in reducing alcohol-related liver damage before the development of alcoholic liver disease. Then at week 4, subjects in the test group showed significantly lower GGT levels $(p=0.017)$ as compared with those in the placebo group.

Higashikawa et al. reported that L. plantarum SN13T improved serum total cholesterol level in healthy adults in normal range [12]. In the test group of this study, se- rum TG levels tended to decrease at weeks 4 and 8 as compared with week 0 . Furthermore, in the male test group, TG levels significantly decreased at week 4 as compared with week 0 . Generally increasing of serum TG is reversible effect of lipid metabolism by alcohol consumption, so serum TG is useful as one of biomarker for chronic alcohol consumption. Regarding with mechanism of increasing TG, Alcohol metabolism contributes to lipid metabolism by the multiple steps; increasing substrate supply, increasing fat esterification to TG. Generally alcohol increases lipolysis of peripheral adipose fat and the altered liver redox state increases fatty acid synthesis. When alcohol and acetaldehyde dehydrogenases catalyze the oxidation of alcohol via acetaldehyde to 
acetate, the cofactor nicotinamide adenine dinucleotide (NAD) is converted to its reduced form NADH. The resulting increase in the ratio of NADH/NAD is responseble for metabolic imbalance and alteration of redox state in hepatocytes. This increase in substrate supply (glycerol and free fatty acids) enhances the rate of esterification of glycerol-3-phosphate. Furthermore alcohol consumption activates the sterol regulatory element-binding protein (SREBP)-1 that induces genes involved in lipid biosynthesis. Conversely, alcohol consumption downregulates the transcription factor peroxisome proliferator-activated receptor- $\alpha$ (PPAR- $\alpha$ ) that regulates enzymes involved in fatty acid oxidation and prevents fatty acid import into the mitochondria [15]. In addition, the prooxidants are generated from alcohol metabolism or from activated phagocytes, resulting lipid peroxidation.

L. brevis SBC8803 could improve the lipid meta- bolism in habitual drinkers. It may be important meaning that the both of GGT and TG increased in this study as the postulated mechanisms. Recently, the meaning of elevated GGT is getting more important for the risks of future developments of liver diseases, type 2 diabetes and cardiovascular disease are increased in several cohort studies when GGT was high [7,8]. In addition, from the biochemical points, the increase of GGT was resulted by the oxidized stresses [9], and the initial manifestation of oxidized stress-induced diseased conditions including alcoholic liver injury, and non-alcoholic steatohepatitis in patients with metabolic syndrome [10]. Therefore, it is getting more important to monitor GGT as the initial manifestation of alcohol- induced organ injury, in addition to monitoring marker for chronic alcohol consumption.

In our previous study involving C57BL/6N mice that had been fed an ethanol-containing diet, L. brevis SBC8803 ameliorated fatty liver and ethanol-induced liver injury by suppressing the upregulation of TNF- $\alpha$ as well as SREBP-1 and SREBP-2 in the liver [13]. Alcohol increases SREBP-1 activity by decreasing the activities of AMP-activated protein kinase and sirtuin-1; TNF- $\alpha$ produced in response to alcohol exposure causes fatty liver by upregulating SREBP-1 activity $[15,16]$. We speculate that $L$. brevis SBC8803 also may influence the regulation of TNF- $\alpha$ and SREBPs in habitual drinkers without obvious hepatocytes damage by alcohol, following the changing of lipid metabolism and oxidized stress condition.

In this study, the preferable effect of lactic acid bacteria for serum GGT and TG seemed to be felt in the 4th week. We have to consider compliance of administration in habitual drinkers for more suitable amount per administration and number of uses. Additionally the storage condition of probiotics might be important. The capsules for eight weeks were packed in an aluminum pouch. The live $L$. brevis of capsules used in this study were kept though the trail in our laboratory. However, there is still a possibility that live L. brevis decreased because of change of temperature by movement of pouch of capsules from the refrigerator at the intake time. We have a speculation that the decrease of live lactic acid bacteria might influence the efficacy of capsules in 8 weeks period. Therefore, we started the improvement of the technology for storing live $L$. brevis until use, or an alternative use of killed L. brevis [17] or an isolated active substance, polyphosphate derived from L. brevis [18]. Further studies are needed to adapt these alternatives.

In conclusion, the intake of $L$. brevis SBC8803 may alleviate alcoholic liver damage in humans. Further research is required to clarify the degree of efficacy and the mechanisms by which probiotics affect liver function.

\section{REFERENCES}

[1] World Health Organization, "Global Status Report on Alcohol and Health," 2011.

[2] Ministry of Health, Labour and Welfare, "The National Nutrition Survey in Japan (in Japanese),” 2008.

[3] Ministry of Welfare, "The Alcohol-Related Problems in Japan (in Japanese),” 1993.

[4] S. Stewart and C. Day, “Alcohol and the Liver,” In: J. S. Dooley, A. S. F. Lok, A. K. Burroughs and E. J. Heathcote, Eds., Sherlok's Disease of The Liver and Biliary System, 12th Edition, Wiley-Blackwell, Oxford, 2011, pp. 507-520.

[5] T. Neumann and C. Spies, "Use of Biomarkers for Alcohol Use Disorders in Clinical Practice,” Addiction, Vol. 98, Suppl. 2, 2003, pp. 81-91. doi:10.1046/j.1359-6357.2003.00587.x

[6] V. Tahan, B. Canbakan, H. Balci, F. Dane, H. Akin, G. Can, I. Hatemi, V. Olgac, A. Sonsuz, G. Ozbay, I. Yurdakul and H. Senturk, "Serum Gamma-Glutamyltranspeptidase Distinguishes Non-Alcoholic Fatty Liver Disease at High Risk,” Hepato-Gastroenterology, Vol. 55, No. 85, 2008, pp. 1433-1438.

[7] C. Meisinger, H. Löwel, M. Heier, A. Schneider and B. Thorand, "KORA Study Group. Serum Gamma-Glutamyltranferase and Risk of Type 2 Diabetes Mellitus in Men and Women from the General Population,” Journal of Internal Medicine, Vol. 258, No. 6, 2005, pp. 527-535. doi:10.1111/j.1365-2796.2005.01572.x

[8] E. Ruttmann, L. J. Brant, H. Concin, G. Diem, K. Rapp and H. Ulmer, Vorarlberg Health Monitoring and Promotion Program Study Group, “Gamma-Glutamyltransferase as a Risk Factor for Cardiovascular Disease Mortality: An Epidemiological Investigation in a Cohort of 163,944 Austrian Adults," Circulation, Vol. 112, No. 14, 2005, pp. 2130-2137.

doi:10.1161/CIRCULATIONAHA.105.552547

[9] D. H. Lee, R. Blomhoff and D. R. Jacobs, "Is Serum 
Gamma Glutamyltransferase a Marker of Oxidative Stress?” Free Radical Research, Vol. 38, No. 6, 2004, pp. 535-539. doi:10.1080/10715760410001694026

[10] A. O. Rantala, M. Lilja, H. Kauma, M. J. Savolainen, A. Reunanen and Y. A. Kesäniemi, "Gamma-Glutamyl Transpeptidase and the Metabolic Syndrome,” Journal of Internal Medicine, Vol. 248, No. 3, 2000, pp. 230-238. doi:10.1046/j.1365-2796.2000.00723.x

[11] I. A. Kirpich, N. V. Solovieva, S. N. Leikhter, N. A. Shidakova, O. V. Lebedeva, P. I. Sidorov, T. A. Bazhukova, A. G. Soloviev, S. S. Barve, C. J. McClain and M. Cave, "Probiotics Restore Bowel Flora and Improve Liver Enzymes in Human Alcohol-Induced Liver Injury: A Pilot Study," Alcohol, Vol. 42, No. 8, 2008, pp. 675-682. doi:10.1016/j.alcohol.2008.08.006

[12] F. Higashikawa, M. Noda, T. Awaya, K. Nomura, H. Oku and M. Sugiyama, "Improvement of Constipation and Liver Function by Plant-Derived Lactic Acid Bacteria: A Double-Blind, Randomized Trial,” Nutrition, Vol. 26, No. 4, 2010, pp. 367-374. doi:10.1016/j.nut.2009.05.008

[13] S. Segawa, Y. Wakita, H. Hirata, J. Watari, "Oral Administration of Heat-Killed Lactobacillus brevis SBC8803 Ameliorates Alcoholic Liver Disease in Ethanol-Containing Diet-Fed C57BL/6N Mice,” International Journal of Food Microbiology, Vol. 128, No. 2, 2008, pp. 371377. doi:10.1016/j.ijfoodmicro.2008.09.023
[14] C. S. Lieber, "Alcoholic Fatty Liver: Its Pathogenesis and Mechanism of Progression to Inflammation and Fibrosis," Alcohol, Vol. 34, Vol. 1, 2004, pp. 9-19.

[15] T. M. Donohue, "Alcohol-Induced Steatosis in Liver Cells," World Journal of Gastroenterology, Vol. 13, No. 37, 2007, pp. 4974-4978.

[16] M. You and D. W. Crabb, "Molecular Mechanism of Alcoholic Fatty Liver: Role of Sterol Reguratory Element-Binding Proteins,” Alcohol, Vol. 34, No. 1, 2004, pp. 39-43. doi:10.1016/j.alcohol.2004.07.004

[17] N. Ueno, M. Fujiya, S. Segawa, T. Nata, K. Moriichi, H. Tanabe, Y. Mizukami, N. Kobayashi, K. Ito and Y. Kohgo, "Heat-Killed Body of Lactobacillus Brevis SBC8803 Ameliorates Intestinal Injury in a Murine Model of Colitis by Enhancing the Intestinal Barrier Function," Inframmatory Bowel Disease, Vol. 17, No. 11, 2011, pp. 2235-2250.

[18] S. Segawa, M. Fujiya, H. Konishi, N. Ueno, N. Kobayashi, T. Shigyo T and Y. Kohgo, "Probiotic-Derived Polyphosphate Enhances the Epithelial Barrier Function and Maintains Intestinal Homeostasis through Integrin-p38 MAPK Pathway,” PLoS One, Vol. 6, No. 8, 2011, Article ID: e22378. doi:10.1371/journal.pone.0023278 